\title{
Non-steroidal Anti-inflammatory Drugs in Tonic, Phasic and Inflammatory Mouse Models
}

\author{
Authors \\ Hugo F. Miranda ${ }^{1}$, Viviana Noriega ${ }^{2}$, Fernando Sierralta ${ }^{3}$, Paula Poblete ${ }^{1}$, Nicolas Aranda1, Juan Carlos Prieto ${ }^{2,3}$
}

\section{Affiliations}

1 Neuroscience Department, Faculty of Medicine, Universidad de Chile, Santiago, Chile

2 Cardiovascular Department, Clinical Hospital, Universidad de Chile, Santiago, Chile

3 Pharmacology Program, ICBM, Faculty of Medicine, Universidad de Chile, Santiago, Chile

\section{Key words}

non-steroidal anti-inflammatory drugs, writhing test, tail flick assay, formalin orofacial test

$\begin{array}{ll}\text { received } & 16.04 .2019 \\ \text { accepted } & 06.06 .2019\end{array}$

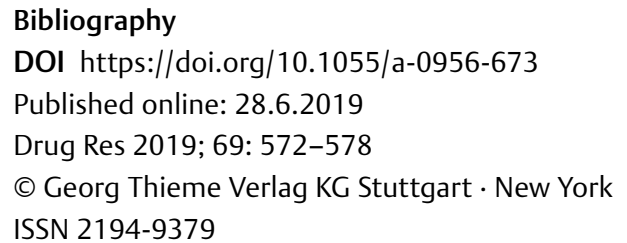

\section{Correspondence}

Hugo F. Miranda

Neuroscience Department, Faculty of Medicine, Universidad de Chile, Independencia 1027 775000 Santiago,

Chile

Tel.: + 56/229/786 237, Fax: + 56/978/6 237

hmiranda@med.uchile.cl

\begin{abstract}
The principal mechanism of action of non-steroidal anti-inflammatory drugs (NSAIDs) is the inhibition of ciclooxigenases. In this study was evaluated if NSAIDs could induce antinociceptive differences according to the type of murine pain model. Male mice were injected intraperitoneally with meloxicam, diclofenac, piroxicam, metamizol, ibuprofen, naproxen and paracetamol in the writhing, tail flick and formalin orofacial tests and dose-response were analyzed to obtain the $\mathrm{ED}_{50}$ of each drugs. Administration of NSAIDs produced in a dose-dependent antinociception with different potency in the tests. The relative potency of NSAIDs among the tests shows a value of 5.53 in the orofacial formalin test in phase I and 6.34 in phase II between meloxicam and paracetamol; of 7.60 in the writhing test between meloxicam and paracetamol and of 8.46 in the tail flick test between ibuprofen and paracetamol. If the comparison is made for each NSAID in the different tests, the minimum value was 0.01 for between writhing and phase II of the orofacial formalin. Meanwhile, the highest power ratio was 11.71 for diclofenac between writhing and tail flick tests. In conclusion, the results suggests that intraperitoneal NSAIDs administration induce antinociceptive activity depending on the type of pain. The results support that NSAIDs administration, induce a wide variety of antinociceptive effect, depending on the type of pain. This suggest the participation of different mechanisms of action that can be added to the simple inhibition of COXs controlled by NSAIDs.
\end{abstract}

\section{Introduction}

The complexity of pain, whether phasic or tonic, has promoted the use of drugs with different mechanisms and sites of antinociceptive activity different mechanisms of action that could contribute to its modulation and consequently to its pharmacotherapy. Among the various types of drugs used for this purpose, the nonsteroidal anti-inflammatory drugs (NSAIDs) should be mentioned since these drugs possess antipyretic, analgesic, and anti-inflammatory properties.

The primary mechanism of action of NSAIDs is the reduction of inflammatory mediators peripherally and centrally by inhibition of cyclooxygenase (COX) enzymes [1]. However, it has been demonstrated that NSAIDs have also other mechanisms of action, between them appear the ability of NSAIDs to penetrate biological membranes where they disrupt important processes of cellular function, action on mircroglial activity, alteration in interleukin production, interfere with L-selectin function [2-4].

Recent advances in the understanding of the different molecular mechanisms of COXs have allowed suggest that NSAIDs are involved in other pharmacological activities, among them which should be highlighted the tumor inhibitions and prevention of metastasis, Alzheimer's and Parkinson's diseases, a key role in bone physiology. [4-6]. 
The pharmacological activity of NSAIDs is based on their ability to inhibit COXs, which provides evidence of their antinociceptive effects that have been validated in several trials, such as the test of hot-plate, the tail-flick, the tail-withdrawal, the radiant heat pawwithdrawal, the von Frey filament, the cold sensitivity (acetone), the abdominal constriction (whrithing) induced by acetic acid (whrithing), formalin oral and paw, the capsaicin, the chronic constriction surgical nerve injury, spared nerve injury, spinal nerve ligation, intraplantar zymosan, intraplantar carrageenan, complete Freund's adjuvant (CFA), lipopolysaccacharide (LPS) tests. The utility of nociception tests is to measure the effectiveness and potency of pain drugs as identifying adverse effects.

Although it has been demonstrated the antinociceptive capacity of NSAIDs, there are no comparative studies of their relative potencies in tonic, phasic and inflammatory pain models. The objective of the present study was to evaluate whether NSAIDs could induce a rank order of potency according to the type of pain model.

\section{Materials and Methods}

\section{Animals}

Male CF-1 mice (25-28g), housed on a $12 \mathrm{~h}$ light-dark cycle at $22 \pm 1{ }^{\circ} \mathrm{C}$ with ccess to food and water ad libitum, were used. All animal procedures were approved by the Animal Care and Use Committee at the Faculty of Medicine, University of Chile (Protocol CBA 0410/ FMUCH2013). Animals were acclimatized to the laboratory for at least $1 \mathrm{~h}$ before testing, used only once during the protocol, and euthanized by overdose of anaesthetic immediately after the algesiometric test with a lethal intraperitoneal (i.p.) injection of $60 \mathrm{mg} / \mathrm{kg}$ of pentobarbital. The number of animals was kept at a minimum, compatible with consistent effects of the drug treatment.

\section{Measurement of antinociceptive activity}

Analgesic activity was assessed by the following test: (A) acetic acid abdominal contraction test (writhing test), as previously described [7]. Antinociception, expressed in \% of maximum possible effect (\% MPE), was calculated as percent inhibition of the saline control writhes $(19.80 \pm 1.45, n=12)$. (B) tail-flick as described previously [8]. Tail flick latencies control were $2.45 \pm 0.08(n=12)$ and converted to \%MPE as follows: \%MPE = (postdrug latency - predrug latency)/(cut-off time - predrug latency) and (C) the orofacial formalin test described previously was used [8]. Total grooming time in each phase was converted to \% MPE as follows: \%MPE $=100-$ (postdrug grooming time/control grooming time saline) $\times 100$.

For each NSAIDs the $D_{50}$, dose that induce $50 \%$ of MPE was calculated from lineal regression of dose-response curves.

\section{Experimental design}

In order to determine the antinociceptive potency of i.p. NSAIDs, dose-response curves produced by meloxicam $(3,10,30$ or $100 \mathrm{mg} /$ $\mathrm{kg})$, naproxen $(3,10,30$ or $100 \mathrm{mg} / \mathrm{kg})$, diclofenac $(1,3,10,30$ or 100 , $\mathrm{mg} / \mathrm{kg}$ ), piroxicam (10,30,60 or $100 \mathrm{mg} / \mathrm{kg})$, metamizol $(3,10,30,100$ or $300 \mathrm{mg} / \mathrm{kg})$, ibuprofen $(3,10,30$ or $100 \mathrm{mg} / \mathrm{kg}$ ) and paracetamol $(3,10,30$, or $100 \mathrm{mg} / \mathrm{kg})$ were obtained in the writhing, tail flick and orofacial formalin tests using at least 6 animals for each at least 4 doses.

\section{Drugs}

Drugs were freshly dissolved in sterile physiological salt solution of $10 \mathrm{~mL} / \mathrm{Kg}$, for intraperitoneal. Paracetamol was provided by Bristol-Myers-Squibb, meloxicam, metamizol and naproxen by Saval Laboratories Chile, ketoprofen by Rhone-Poulenc Rorer, piroxicam and parecoxib by Pfizer Chile, diclofenac by Novartis Chile S.A. and ibuprofen by Laboratorio Chile.

\section{Statistical analysis}

Results are presented as means \pm SEM. The statistical difference between NSAIDs was assessed by one-way ANOVA, followed by Tukey's post test for and $p$ values less than 0.05 ( $p<0.05$ ) were considered statistically significant. Statistical analyses were carried out using the program Pharm Tools Pro, version 1.27, McCary Group Inc., PA, USA.

\section{Results}

It is noteworthy that the doses of NSAIDs used in the present work did not produce significant changes in the comportment or the motor activity of the animals.

\section{Antinociception induced by NSAIDs in the acetic acid writhing test}

Administration of solution of acetic acid via i.p. produced nociception characterized by abdominal contraction which were dose-dependent reduced by the diverse doses of NSAIDs, with different potencies as shown in > Table $\mathbf{1}$ and $>$ Fig. $\mathbf{1}$.

- Table $1 \mathrm{ED}_{50}$ values with SEM (mg/kg, i.p.) for the antinociceptive effect of NSAIDs In the acetic acid writhing (wt), tall flick (TF), and phase I and phase II of the formalin orofacial tests of mice.

\begin{tabular}{|l|c|c|c|c|}
\hline \multirow{2}{*}{ Drugs } & \multicolumn{4}{|c|}{ ED50 \pm SEM (mg/kg i.p.) } \\
\cline { 2 - 5 } & WT & TF & OF I & OF II \\
\hline Meloxicam & $6.50 \pm 0.54$ & $73.22 \pm 7.65$ & $8.06 \pm 0.88$ & $6.65 \pm 0.54$ \\
\hline Diclofenac & $7.20 \pm 0.90$ & $84.34 \pm 5.12$ & $13.54 \pm 2.06$ & $31.23 \pm 5.65$ \\
\hline Piroxicam & $8.50 \pm 1.20$ & $21.54 \pm 2.51$ & $33.56 \pm 3.21$ & $42.21 \pm 6.99$ \\
\hline Metamizol & $28.50 \pm 3.17$ & $117.19 \pm 13.90$ & $36.56 \pm 6.59$ & $18.25 \pm 3.10$ \\
\hline Ibuprofen & $33.95 \pm 1.93$ & $14.66 \pm 2.03$ & $39.68 \pm 3.96$ & $35.59 \pm 3.98$ \\
\hline Naproxen & $46.76 \pm 3.40$ & $87.46 \pm 10.78$ & $9.67 \pm 2.00$ & $17.70 \pm 2.13$ \\
\hline Paracetamol & $49.46 \pm 3.31$ & $124.05 \pm 15.70$ & $44.63 \pm 4.78$ & $37.37 \pm 4.05$ \\
\hline
\end{tabular}



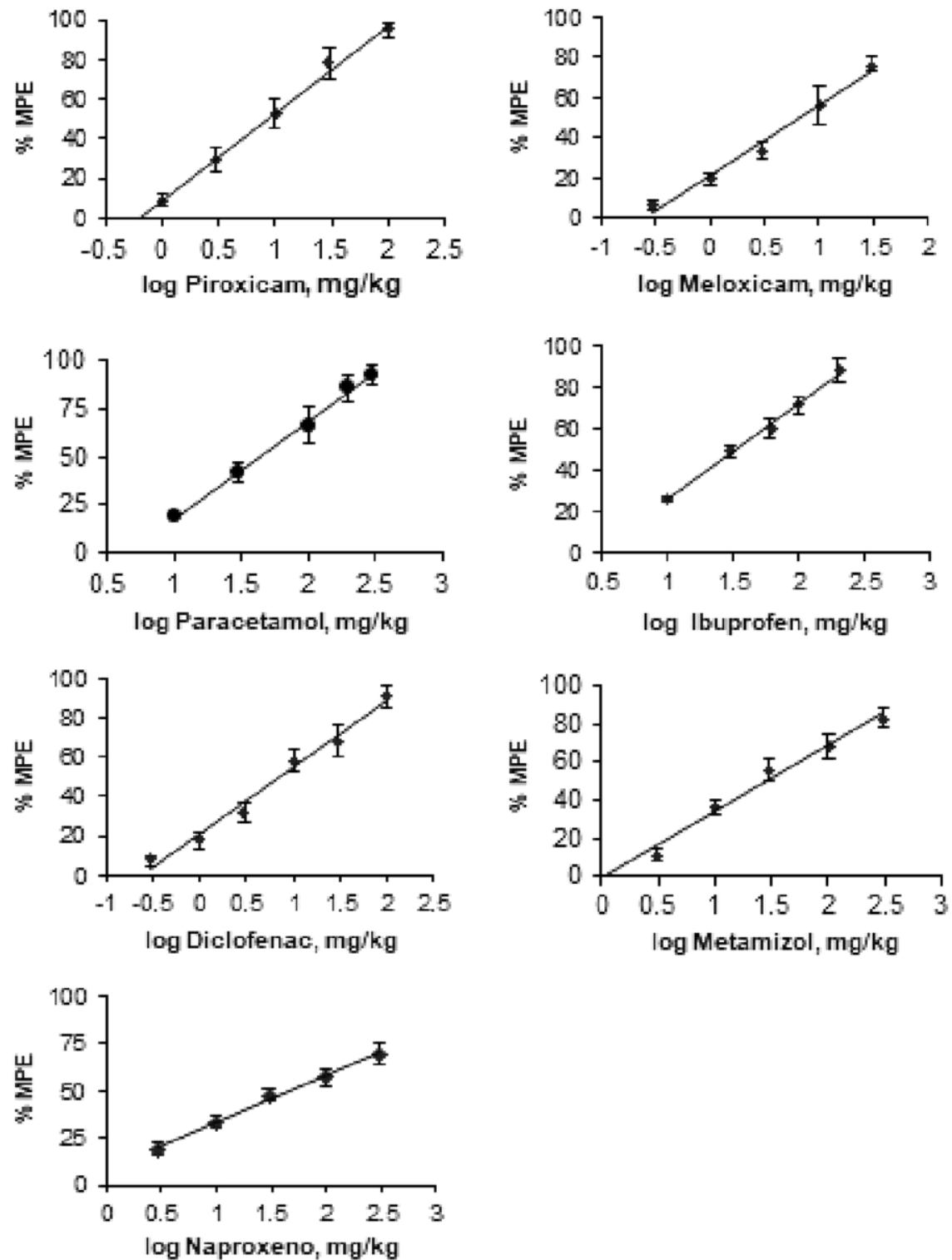

-Fig. 1 Dose-response curves for the antinociceptive effect in mice induced by i.p. NSAIDs in the writhing test . Each point is the means \pm SEM of 6-8 animals. \% MPE = antinociception represented as percentage of maximum possible effect.

Furthermore, the $\mathrm{ED}_{50}$ values demonstrated the following rank order of potency of writhes inhibition by NSAIDs was: meloxicam $=$ diclofenac $>$ piroxicam $>$ metamizol $>$ ibuprofen $>$ naproxen $=$ paracetamol.

\section{Antinociception induced by NSAIDs in the tail flick test}

The i.p. administration of the different doses of NSAIDs used in this work produced a dose-related antinociceptive activity but with diverse potencies in this test, see $>$ Table $\mathbf{1}$ and $>$ Fig. $\mathbf{2}$.

Also, the rank order of potency of NSAIDs, according the $\mathrm{ED}_{50}$ values, in this test was: ibuprofen $>$ piroxicam $>$ meloxicam $>$ diclofenac $=$ naproxen $>$ metamizol $>$ paracetamol.

\section{Antinociception induced by NSAIDs in the formalin orofacial test}

After i.p. administration of different doses of NSAIDs a dose-related antinociceptive response was obtained in phase I and phase II of the orofacial formalin assay, characterized by the difference in its potency, as it can be seen in > Table $\mathbf{1}$ and $>$ Fig. $\mathbf{3}$.

In this assay, the rank of potency of NSAIDs, measured by the $\mathrm{ED}_{50}$ values, was: meloxicam $>$ naproxen $>$ diclofenac $>$ piroxi$\mathrm{cam}=$ metamizol $=$ ibuprofen $>$ paracetamol. 

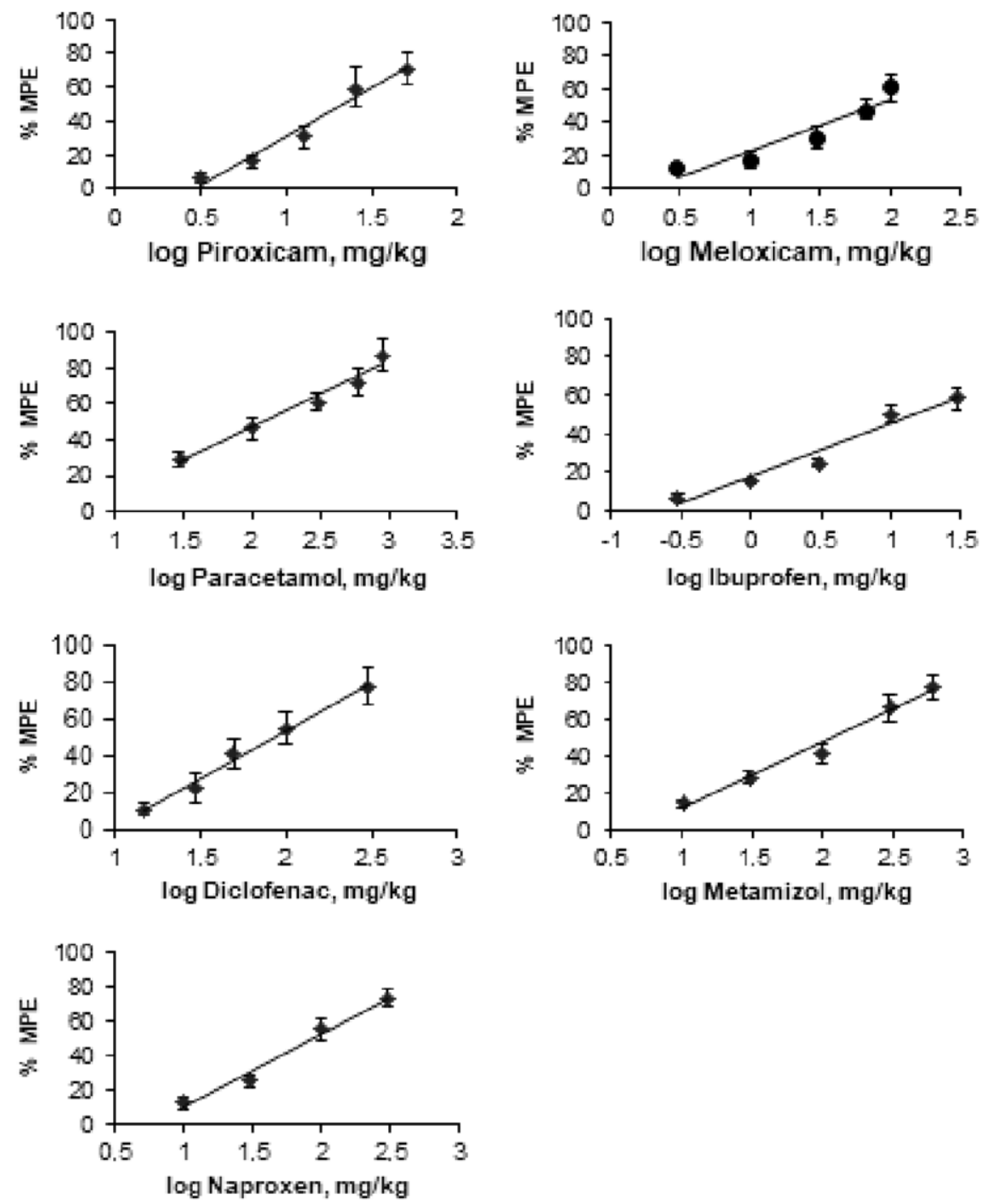

- Fig. 2 Dose-response curves for the antinociceptive effect in mice induced by i.p. NSAIDs in the tail flick test. Each point is the means \pm SEM of 6-8 animals. \% MPE = antinociception represented as percentage of maximum possible effect.

\section{Antinociceptive potency of NSAIDs in the diverse tests}

The analysis of the relative potency of NSAIDs among the tests studied shows a value of 5.53 in the orofacial formalin test in phase I and 6.34 in phase II between meloxicam and paracetamol; of 7.60 in the writhing test between meloxicam and paracetamol and of 8.46 in the tail flick test between ibuprofen and paracetamol.

If the comparison is made for each NSAID in the different tests used, the minimum value was 0.01 for between writhing and phase II of the orofacial formalin. Meanwhile, the highest power ratio was 11.71 for diclofenac between writhing and tail flick tests. All values of comparative NSAIDs potency ratio are shown in > Fig. 4.

\section{Discussion}

The findings of the present study demonstrated that the following NSAIDs: diclofenac, ibuprofen, meloxicam, metamizol, naproxen, paracetamol and piroxicam possesses an important activity antinociceptive, independent of the noxious stimulus. In this occasion, the tests used were tonic pain (acetic acid writhing test), phasic pain (tail flick test) and inflammatory pain (orofacial formalin test). Furthermore, results obtained are in agreement with previous reported different profiles of nociceptive activity of NSAIDs in algesiometer tests [7-12].

The analysis of the results obtained in the assay of abdominal contraction by acetic acid or writhing test, shows that COX- 1 inhibitor NSAIDs are more potent than COX-2 inhibitors, with the exception of meloxicam and that they also possess greater potency than 

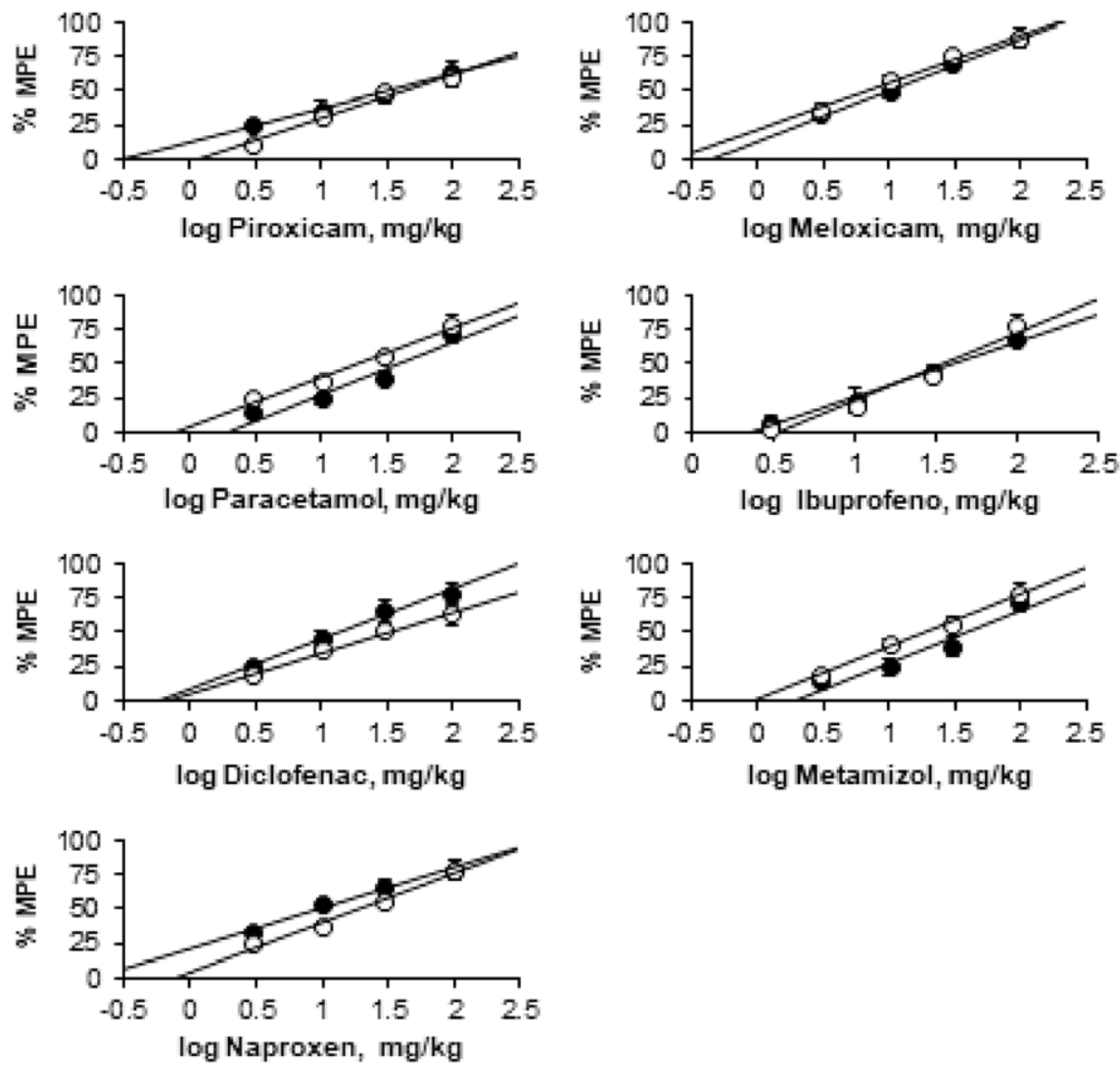

- Fig. 3 Dose-response curves for the antinociceptive effect in mice induced by i.p. NSAIDs in the orofacial test phase in I (•) and phase II (o). Each point is the means \pm SEM of 6-8 animals. \% MPE = antinociception represented as percentage of maximum possible effect.

those that are ascribed as COX-3 inhibitors. Besides, the findings obtained in the tail flick and the formalin orofacial tests, displays the similar order the potency that in the writhing test. These findings demonstrate the ability of NSAIDs to produce antinociception in murine models of tonic, phasic and inflammatory pain.

The results of this study show that meloxicam was found to be more potent than other NSAIDs used in tonic and inflammatory pain models, not in phasic pain. In addition, in all the tests used, paracetamol was the weakest. Generalizing, COX-1 and COX-2 inhibitors NSAIDs were the most effective and the NSAID related to COX-3, paracetamol, was the least effective.

It is well supported that several mediators are implicated in the modulation of pain allowing various probable new aims for pharmacotherapy. According to this hypothesize, it has been established that the mechanism by which NSAIDs induce antinociception is mostly by inhibiting COXs and the concentration of prostaglandins, both centrally and peripherally. However, the existence of other mechanisms that could explain its therapeutic effects has been demonstrated.

Thereby, among the evidences has been included their interaction with monoaminergic, nitric oxide, endocannabinoids, serotonergic and cholinergic systems and endogenous opioid pathway [13-16].
On the other hand, recent investigations have proposed other mechanisms of action for NSAIDs, between them inhibition of prostaglandin keto reductase (PTGR) enzymes responsible for the inactivation of prostaglandins. Modulation of lactoferrin (LF) and transthyretin (TTR), transporting proteins for NSAIDs reducing concentration of the drugs in the body and the action of phospholipase (PLA) which suspends the production of arachidonic acid from phospholipids. [17-19].

Furthermore, other evidence has been reported anti-inflammatory actions of NSAIDs though COX-independent, among which should be mentioned that they are able to induce the downregulation of L-selectin, inhibition of nuclear factor kappa B, including the proinflammatory cytokines such as TNF- $\alpha$ or IL- $1 \beta$, inhibition of activity of i-NOS [20-22].

The results presented support that NSAIDs administration, induce a wide variety of antinociceptive effect, depending on the type of pain. This antinociceptive activity suggest the participation of different mechanisms of action that can be added to the simple inhibition of COXs controlled by NSAIDs.

\section{Conclusions}

The current data support that intraperitoneally application of NSAIDs have antinociceptive activity and that this effect appears 

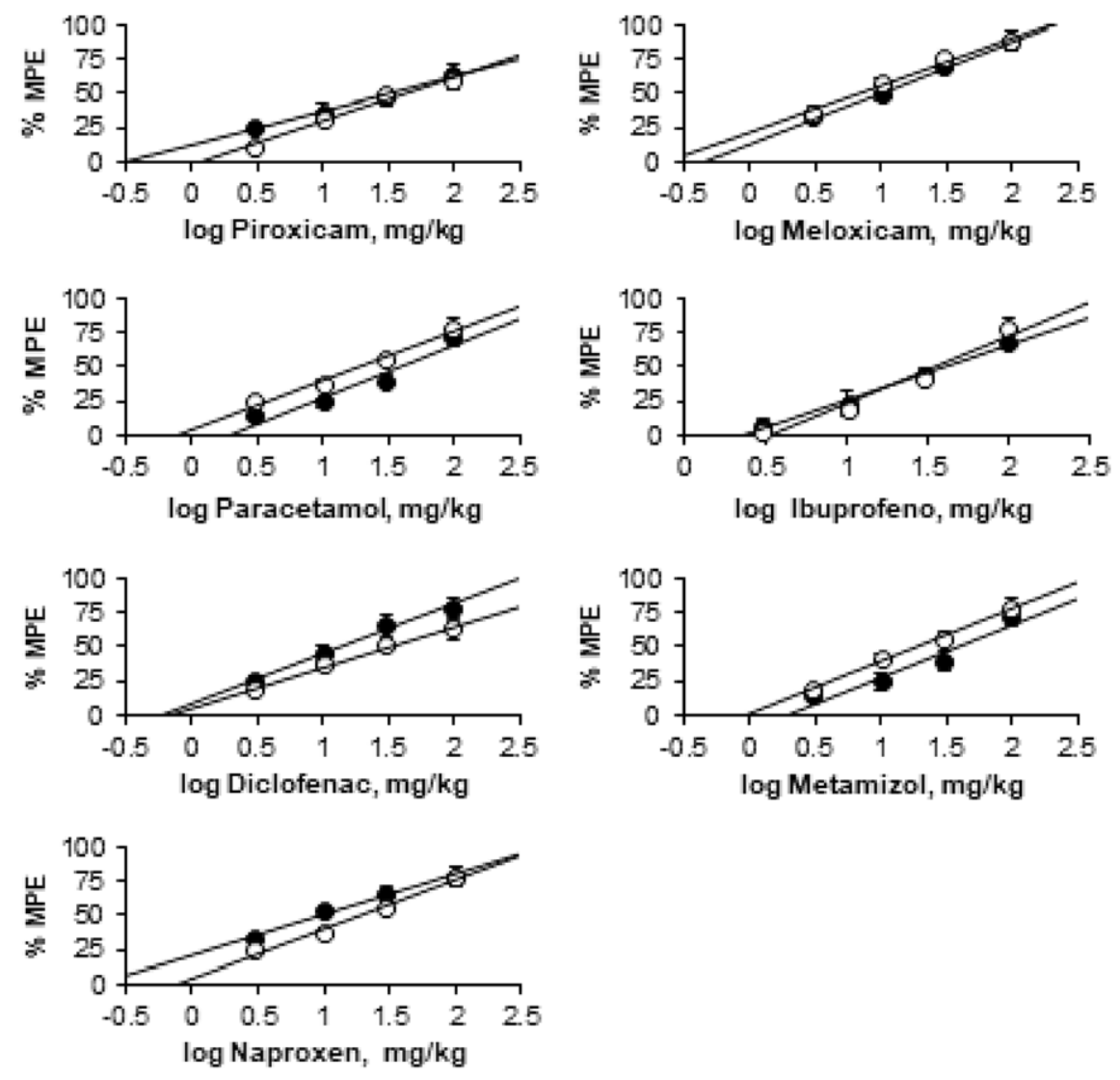

-Fig. 4 Ratio of relative potency of NSAIDs among the algesiometer tests. Writhing test (WT), tail flick test (TF) and formalin orofacial test, phase I (OF I) and phase II (OF II).

to be mediated by mechanisms of action further that simply COX inhibition.

\section{Conflict of Interest}

The authors declare that they have no competing interest related to this study

\section{References}

[1] Smith WL, DeWitt DL, Garavito RM. Cyclooxygenases: Structural, cellular, and molecular biology. Ann Rev Biochem 2000; 69: 14501820

[2] Wong RSY. Role of Nonsteroidal Anti-Inflammatory Drugs (NSAIDs) in Cancer Prevention and Cancer Promotion. Adv Pharmacol Sci 2019; 3418975. doi:10.1155/2019/3418975

[3] Gan TJ. Diclofenac: An update on its mechanism of action and safety profile. Curr Med Res Opin 2010; 26: 1715-1731

[4] Diaz-Gonzalez F, Sanchez-Madrid F. NSAIDs: Learning new tricks from old drugs. Eur. J. Immunol 2015; 45: 679-686

[5] Gunaydin C, Sirri Bilge S. Effects of Nonsteroidal Anti-Inflammatory drugs at the molecular level. Eurasian J Med 2018; 50: 116-121
[6] Lisowska B, Kosson D, Domaracka K. Lights and shadows of NSAIDs in bone healing: The role of prostaglandins in bone metabolism. Drug Des Devel Ther 2018; 12: 1753-1758

[7] Pinardi G, Sierralta F, Miranda HF. Atropine reverses the antinociception of nonsteroidal anti-inflammatory drugs in the tail-flick test of mice. Pharmacol Biochem Behav 2003; 74: 603-608

[8] Miranda HF, Puig MM, Prieto JC et al. Synergism between paracetamol and nonsteroidal anti-inflammatoy drugs in experimental acute pain. Pain 2006; 121: 22-28

[9] Miranda HF, Sierralta F, Prieto JC. Synergism between NSAIDs in the orofacial formalin test in mice. Pharmacol Biochem Behav 2009; 92: 314-318

[10] Muñoz ], Navarro C, Noriega $V$ et al. Synergism between COX-3 inhibitors in two animal models of pain. Inflammopharmacol 2010; 18: 65-71

[11] Miranda HF, Noriega V, Zepeda RJ et al. Systemic synergism between codeine and morphine in three pin models in mice. Pharmacol Rep 2013; 65: 80-88

[12] Miranda HF, Sierralta F, Aranda N et al. Pharmacological profile of dexketoprofen in orofacial pain. Pharmacol Rep 2016; 68: 1111-1114

[13] Hamza M, Dionne RA. Mechanisms of non-opioid analgesics beyond cyclooxygenase enzyme inhibition. Curr Mol Pharmacol 2009; 2: 1-14

[14] Isiordia-Espinoza MA, Pozos-Guillén A, Pérez-Urizar ] et al. Involvement of nitric oxide and ATP-sensitive potassium channels in the peripheral antinoceptive action of a tramadol-dexketoprofen combination in the formalin test. Drug Dev Res 2014; 75: 449-454 
[15] Miranda HF, Sierralta F, Aranda N et al. Pharmacological profile of dexketoprofen in orofacial pain. Pharmacol Rep. 2016; 68: 1111-1114

[16] Raffa RB, Stone DJ Jr, Tallarida RJ. Discovery of 'self-synergistic' spinal/ supraspinal antinociception produced by acetaminophen (paracetamol). J Pharmacol Exp Ther 2000; 295: 291-294

[17] Singh N, Jabeen T, Sharma S et al. Specific binding of non-steroidal anti-inflammatory drugs (NSAIDs) to phospholipase A2: Structure of the complex formed between phospholipase A2 and diclofenac at 2.7 A resolution. Acta Crystallogr D Biol Crystallogr 2006; 62: 410-416

[18] Wu YH, Ko TP, Guo RT et al. Structural basis for catalytic and inhibitory mechanisms of human prostaglandin reductase PTGR2. Structure 2008; 16: 1714-1723
[19] Dwivedi AK, Gurjar V, Kumar S et al. Molecular basis for nonspecificity of nonsteroidal anti-inflammatory drugs (NSAIDs). Drug Discov Today 2015; 20: 863-873

[20] Herrera-Garcia A, Dominguez-Luis MArce-Franco et al. In vivo modulation of the inflammatory response by nonsteroidal anti-inflammatory drug-related compounds that trigger L-selectin shedding. Eur J Immunol 2013; 43: 55-64

[21] Barnes P], Karin M. Nuclear factor-kappaB: A pivotal transcription factor in chronic inflammatory diseases. N Engl J Med 1997; 336: 1066-1071

[22] Dıaz-Gonzalez F, Sanchez-Madrid F. NSAIDs: Learning new tricks from old drugs. Eur J Immunol 2015; 45: 679-686 